\title{
Lineage and Birth Date Specify Motor Neuron Targeting and Dendritic Architecture in Adult Drosophila
}

\author{
Myungin Baek ${ }^{1}$ and Richard S. Mann ${ }^{2}$ \\ Departments of ${ }^{1}$ Biological Sciences and ${ }^{2}$ Biochemistry and Molecular Biophysics, Columbia University, New York, New York 10032
}

Locomotion in adult Drosophila depends on motor neurons that target a set of multifibered muscles in the appendages. Here, we describe the development of motor neurons in adult Drosophila, focusing on those that target the legs. Leg motor neurons are born from at least 11 neuroblast lineages, but two lineages generate the majority of these cells. Using genetic single-cell labeling methods, we analyze the birth order, muscle targeting, and dendritic arbors for most of the leg motor neurons. Our results reveal that each leg motor neuron is born at a characteristic time of development, from a specific lineage, and has a stereotyped dendritic architecture. Motor axons that target a particular leg segment or muscle have similar dendritic arbors but can derive from different lineages. Thus, although Drosophila uses a lineage-based method to generate leg motor neurons, individual lineages are not dedicated to generate neurons that target a single leg segment or muscle type.

\section{Introduction}

Animal locomotion requires the coordinated excitation of muscles by motor neurons that integrate sensory and interneuron inputs and, as a result, trigger muscle contractions. A common feature of all known motor systems is that the targeting of motor neurons in the periphery has an orderly and stereotyped representation in the CNS. In the chick and mouse, motor neurons that extend into the limbs are located in the lateral motor column, within which are specialized pools of motor neurons that innervate specific limb muscles (Landmesser, 1978; Tsuchida et al., 1994; Jessell, 2000). In the motor system of Drosophila larvae, the position of motor neuron dendritic fields, not their cell bodies, are grouped together according to the muscles that they innervate, generating a myotopic map in the CNS (Landgraf et al., 2003). In Caenorhabditis elegans, the position of motor neuron cell bodies and processes are also highly stereotyped (Sulston, 1976; Sulston and Horvitz, 1977).

Although all of these motor systems exhibit some form of topographic map, there are significant differences in how they develop. In C. elegans, which has the simplest organization, motor neurons are born from precisely defined lineages that are not dedicated to neurogenesis (Sulston and Horvitz, 1977; Sulston, 1983). In insects such as Drosophila, lineage also plays an important role in the generation of neurons, but in this case neurons are generated by neuroblasts (NBs), stem cells that are dedicated to producing neurons and glia (Truman and Bate, 1988; Prokop and Technau, 1991; Broadus et al., 1995; Doe and Skeath, 1996). In

Received April 2, 2009; accepted April 16, 2009.

This work was supported by grants from Project ALS and The Motor Neuron Center of Columbia University. We thank the following people for providing reagents and/or advice during the course of this project: T. Akay, W. Grueber, T. Jessell, B. McCabe, G. Struhl, J. Truman, and D. Williams. We thank T. Akay, W. Grueber, 0. Hobert, T. Jessell, and C. Mendes for comments on this manuscript.

Correspondence should be addressed to Richard S. Mann, Department of Biochemistry and Molecular Biophysics, Columbia University, 701 West 168th Street, HHSC 1104, New York, NY 10032. E-mail: rsm10@columbia.edu. D0I:10.1523/JNEUROSCI.1585-09.2009

Copyright $\odot 2009$ Society for Neuroscience $\quad$ 0270-6474/09/296904-13\$15.00/0 contrast, cell lineage is thought to play no role in the generation of vertebrate motor neurons (Leber et al., 1990). In the spinal cord, most motor neurons are born during a single time window in a progenitor domain that is determined by Sonic hedgehog signaling and homeodomain transcription factors (Jessell, 2000).

Unlike their larval counterparts, many adult insects walk using segmented legs that have a well-developed proximodistal (PD) axis. Walking in insects, as in vertebrates, therefore requires the contraction of flexor and extensor-like muscles that must be coordinated between leg joints and between contralateral legs. The adult insect motor circuit has been extensively studied, most notably in the cockroach, locust, and stick insect (Delcomyn, 1989; Burrows, 1992, 1996; Bässler and Büschges, 1998; Tryba and Ritzmann, 2000; Büschges et al., 2008). As in limbed vertebrates, motor neurons exit the CNS and synapse onto specific muscles that control individual leg joints in the ipsilateral limb. Thus, although simpler, the adult insect motor system shares many aspects with vertebrates and must solve problems not faced by the motor systems of animals that crawl or swim. Although we understand certain aspects of adult leg development in Drosophila (Morata, 2001; Estella and Mann, 2008; Estella et al., 2008; McKay et al., 2009), including the organization of a stereotyped set of multifibered muscles in each leg segment (Soler et al., 2004), much less is known about how this adult motor system develops.

As in embryogenesis, adult Drosophila neurons are derived from a characteristic set of NBs present in the CNS (Truman and Bate, 1988; Truman et al., 2004). Apart from a handful of examples in the Drosophila brain (Lee et al., 1999; Jefferis et al., 2001; Ito and Awasaki, 2008), the neurons born from specific larval NBs have not been characterized. Here, we use genetic methods in Drosophila to characterize the development of the adult leg motor neurons. We find that the leg is innervated by $\sim 50$ motor neurons that are born from 11 different NBs. We analyzed the birth dates, muscle targets, and dendritic arbors of these neurons. Together, these data define the developmental history of the adult 
Table 1. Summary of MARCM analysis with Vglut-Gal4 of leg motor neurons ${ }^{a}$

\begin{tabular}{|c|c|c|c|c|c|c|c|c|}
\hline $\begin{array}{l}\text { Lineage } \\
\text { (embryonic } \\
\text { clones) }\end{array}$ & Total \# of MNs & $\begin{array}{l}\text { \# of times } \\
\text { entire lineage } \\
\text { was observed } \\
\text { (\# embryonic } \\
\text { clones) }\end{array}$ & $\begin{array}{l}\text { Latest time } \\
\text { entire lineage } \\
\text { was labeled } \\
\text { (hours AEL) }\end{array}$ & $\begin{array}{l}\text { Cell body posi- } \\
\text { tion relative to } \\
\text { neuropil }\end{array}$ & $\begin{array}{l}\text { Non-MNs } \\
\text { in the clone? }\end{array}$ & $\begin{array}{l}\text { Partial } \\
\text { lineages } \\
\text { observed? }\end{array}$ & $\begin{array}{l}\text { Targeted leg } \\
\text { segments (\#) }\end{array}$ & Notes \\
\hline$A^{b}$ & $28^{c}$ & $69(16)$ & $\leq 48$ & Anterior & No & Yes & Fe (13), Ti (15) & $\begin{array}{l}9 \text { of } 10 \text { embryonic clones also } \\
\text { labeled F or G; may corre- } \\
\text { spond to NB } 15 \text { of Truman et } \\
\text { al. (2004) }\end{array}$ \\
\hline B & 7 & $47(11)$ & $\leq 54$ & Anterior & No & Yes & $\mathrm{Fe}(1), \operatorname{Tr}(2), \mathrm{Co}(4)$ & \\
\hline C & 1 & $18(2)$ & $\leq 54$ & Posterior & No & NA & $\mathrm{Co}$ & \\
\hline D & 2 & $77(8)$ & $\leq 66$ & Anterior & No & Yes & $\mathrm{Co}(2)$ & \\
\hline$E$ & 1 & $54(10)$ & $\leq 48$ & Posterior & $\sim 25$ inter-neurons & NA & $\mathrm{C}_{0}$ & \\
\hline $\mathrm{F}^{b}$ & 1 & $12(5)$ & $\leq 78$ & Anterior & No & NA & $\mathrm{Co}$ & $\begin{array}{l}\text { Some single-cell clones project } \\
\text { to coxa and femur }\end{array}$ \\
\hline $\mathrm{G}^{b}$ & 1 & $32(5)$ & $\leq 64$ & Anterior & No & NA & $\mathrm{Tr}$ & \\
\hline $\mathrm{H}$ & 2 & $2(1)$ & $\leq 24$ & Anterior & No & NA & $\mathrm{Fe}(2)$ & Embryonic born MN \\
\hline I & 2 & $1(1)$ & $\leq 12$ & Anterior & No & NA & $\mathrm{Fe}(2)$ & Embryonic born MN \\
\hline J & 1 & $1(1)$ & $\leq 12$ & Posterior & No & NA & $\mathrm{Co}$ & Embryonic born MN \\
\hline K & 1 & $1(1)$ & $\leq 12$ & Anterior & No & NA & $\mathrm{Fe}$ & Embryonic born MN \\
\hline \multicolumn{9}{|c|}{ Other MNs (only postembryonic labeling) } \\
\hline V & 1 & $7(0)$ & $\leq 78$ & Anterior & No & NA & $\mathrm{Fe}$ & \\
\hline W & 1 & $2(0)$ & $\leq 76$ & Anterior & No & NA & $\mathrm{Fe}$ & \\
\hline$X$ & 1 & $2(0)$ & $\leq 86$ & Anterior & No & NA & $\mathrm{Fe}$ & \\
\hline Y & 1 & $2(0)$ & $\leq 78$ & Anterior & No & NA & $\mathrm{Fe}$ & \\
\hline
\end{tabular}

${ }^{a}$ Two Vglut-Gal4 ${ }^{+}$motor neurons (MNs) were never observed in the MARCM analyses (one in coxa, one in trochanter). For those lineages that were only observed once (I-K), we cannot rule out that these neurons are part of another lineage. ${ }^{b}$ For Lin A, F, and G, the entire lineage can be labeled by both embryonic and postembryonic clones without labeling any additional motor neuronss, arguing that all three are independent lineages.

'Twenty-seven of the 28 Lin A motor neurons were observed as single-cell clones. The single unlabeled Lin A motor neuron has a very distinctive axon targeting the ventral tibia.

leg motor neurons and show that, although lineage plays an important role in the generation of these neurons, most lineages are not dedicated to give rise only to motor neurons.

\section{Materials and Methods}

Fly stocks. Unless otherwise noted, fly stocks were obtained from the Bloomington Stock Center: yw hs-flp tubGal80 FRT19A; w flp122 FRT19A; vesicular glutamate transporter-galactosidase-4 (Vglut-Gal4) (also called OK371-Gal4) (Mahr and Aberle, 2006); upstream activating sequence (UAS)-CD8/green fluorescent protein (CD8GFP) (on II and III); and myosin heavy chain (MHC)-tauGFP (Soler et al., 2004).

Mosaic analysis with a repressible cell marker analysis. To positively label motor neurons in the adult, flies of the genotype $y w$ hs-flp tubGal80 FRT19A; Vglut-Gal4, UAS-CD8GFP; UAS-CD8GFP were crossed to $y w$ flp122 FRT19A; Vglut-Gal4, UAS-CD8GFP; UAS-CD8GFP flies. Heat shocks to induce clones were given for $60 \mathrm{~min}$ at $37^{\circ} \mathrm{C}$. For embryonic clones, heat shocks were given to $0-12 \mathrm{~h}$ embryos or 12-24 h embryos. For clones induced during larval stages, we varied the heat shock time every $2 \mathrm{~h}$ from 24 to $120 \mathrm{~h}$. To accurately time the heat shocks given during the second and third instar [ $\geq 48 \mathrm{~h}$ after egg laying (AEL)], the morphologies of the larval mouth parts and spiracles present at the first larval molt (at $48 \mathrm{~h} \mathrm{AEL}$ ) were used to set the larval age at $48 \mathrm{~h}$.

Sample preparation and microscopy. Adult flies that had green fluorescent protein (GFP)-positive cells in the T1 CNS were preselected using a dissecting microscope. T2 and T3 legs, head, and abdominal segments were removed, and the remaining tissue was fixed in $4 \%$ paraformaldehyde in PBS overnight at room temperature. The tissue was washed once with PBS and dissected in Vectashield (Vector Laboratories). Dissected CNS and T1 legs from the same fly were mounted together on the same slide, to correlate the two patterns. Multiple 2- $\mu$ m-thick sections in the $z$-axis (dorsoventral for the CNS and mediolateral for the adult leg) were imaged with a Leica TCS SP5 confocal microscope.

Most ( 408 of 428) of the clones derived from the postembryonic heat shocks that we analyzed were composed of only a single motor neuron innervating a $\mathrm{T} 1$ leg; occasionally flies with two (17) or, more rarely, three (3) labeled motor neurons were analyzed (supplemental Fig. 5, available at www.jneurosci.org as supplemental material) (see Fig. 3C-E). Clones were classified as follows. (1) Motor neurons innervating a particular leg segment were grouped together. (2) Within these groups, motor neurons were organized according to which muscle they innervated. The targeted muscle was determined by the position of the axon arbors in the leg and identifying them in preparations of UAS-CD8RFP; Vglut-Gal4 UASCD8RFP; MHC-tauGFP legs. (3) Motor neurons that targeted the same muscle were further classified according to the details of their axon termini (shape, PD position along the muscle, and number of branches). In nearly all cases, these three criteria were sufficient to unambiguously distinguish individual motor neurons. For those cases in which these criteria were insufficient to distinguish motor neurons, the threedimensional patterns of dendritic arbors in the T1 neuromere, analyzed by examining $\sim 40$ confocal sections taken along the dorsoventral axis, were used to classify motor neurons. The results of this classification procedure are summarized in Table 1.

Image analysis. To image motor neuron dendrites in the neuropil, ventral and dorsal Z-stacks, each containing $\sim 20$ sections, were generated using NIH Image J. Binary images for Z-stack images were generated using Photoshop (Adobe Systems). Z-stack images were then aligned based on the structure of the T1 neuromere. Nonmotor neuron branches were removed to avoid including them in the analysis. Heat maps (see Fig. $7 B, C$ ) were generated in Photoshop and represent the degree of overlap in the dendrites for a subset of neurons: green represents single dendrites (i.e., no overlap), and blue represents complete overlap within the set being analyzed.

For the eight sector analyses, the ventral and dorsal sets of Z-stack images for each sample were divided into four sectors (V1 to V4 and D1 to D4) (see Fig. 7A). For each sample, the number of pixels was calculated using NIH Image J for each of the eight sectors. The average pixel intensity was calculated for all motor neurons for each sector. The relative pixel intensity was then calculated for each motor neuron relative to this average. The data are represented in bar graphs (see Fig. $7 B, C$ ) or heat maps (see Fig. $7 D, E$ ) in which green is $\leq 0.1 \times$ relative to the average (underrepresented) and red is $\geq 2 \times$ relative to the average (overrepresented). Error bars in the bar graphs indicate the range of values within the set of neurons being analyzed.

The dendrograms shown in Figure 7, $D$ and $E$, were generated using 

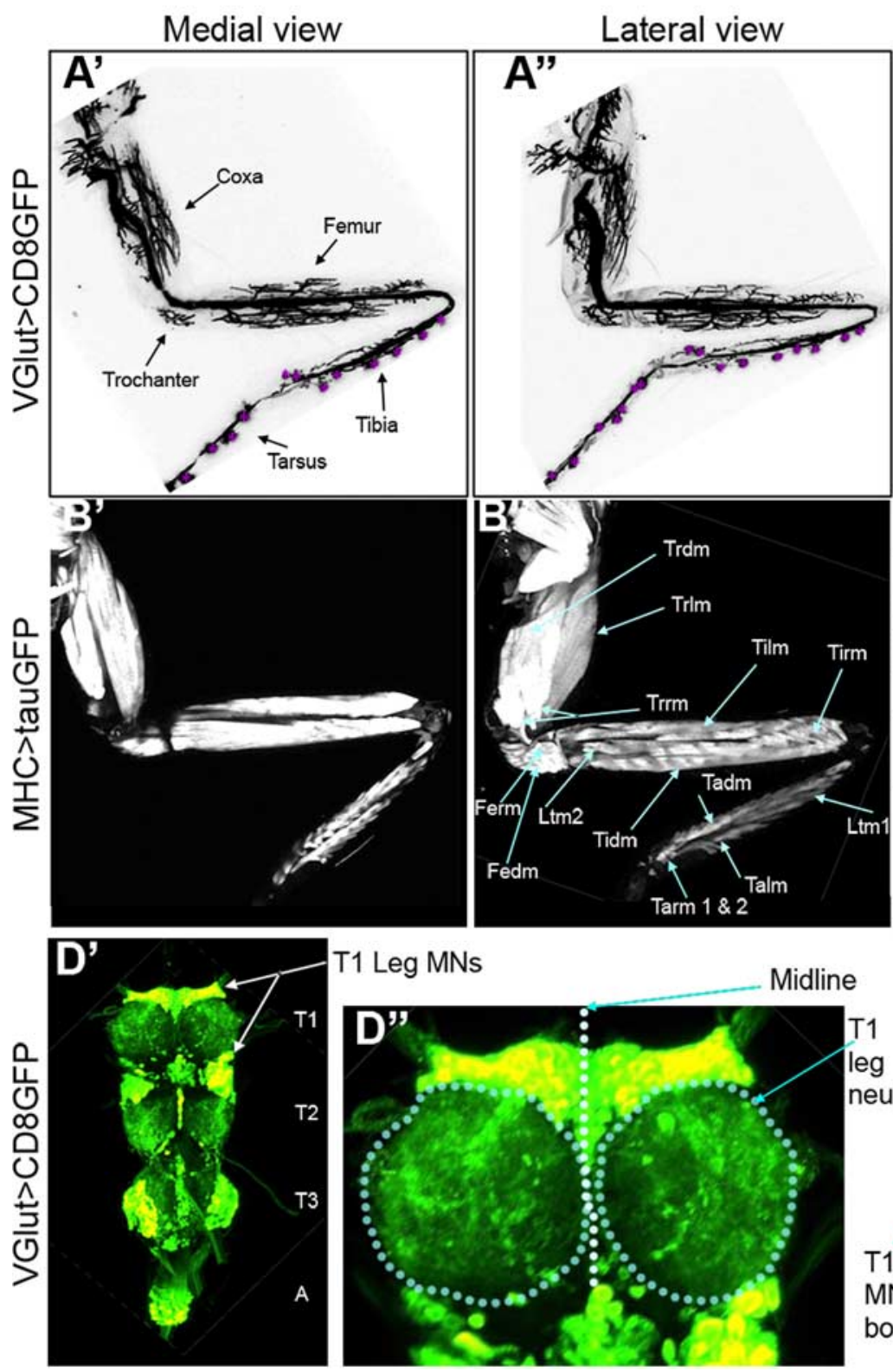

T1 Leg MNs

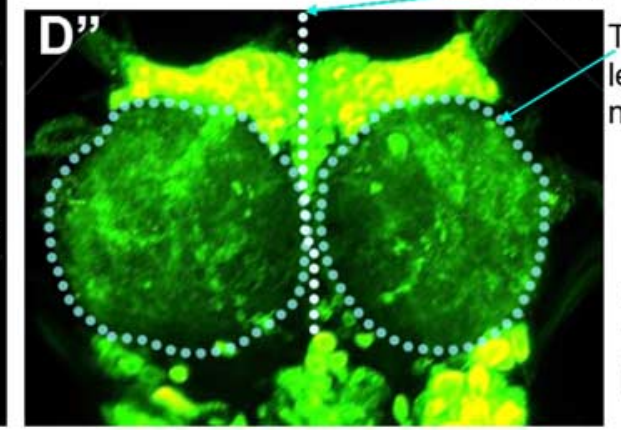

Midline
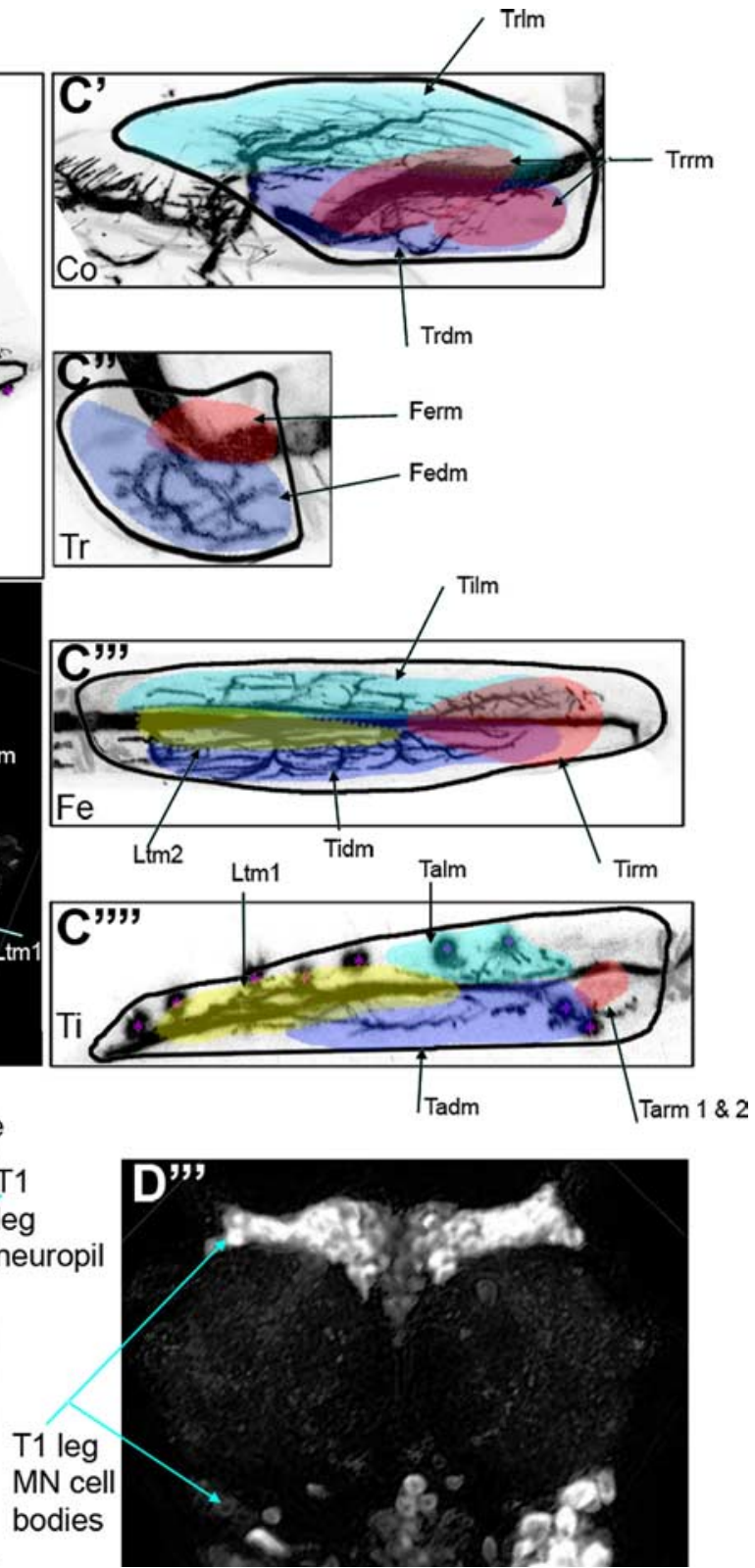

Figure 1. Vglut-Gal4 and MHC-GFP expression patterns. $\boldsymbol{A}$, Adult legs of Vglut-Gal4; UAS-CD8GFP animals imaged for GFP fluorescence. $\boldsymbol{A}^{\prime}$ is a medial view; $\boldsymbol{A}^{\prime \prime}$ is a lateral view. $\boldsymbol{B}$, Adult legs of MHC-tauGFP animals imaged for GFP fluorescence. $\boldsymbol{B}^{\prime}$ is a medial view; $\boldsymbol{B}^{\prime \prime}$ is a lateral view. The muscles are labeled as described previously (Soler et al., 2004). $\boldsymbol{C}$, Higher-magnification views of the individual leg segments from Vglut-Gal4; UAS-CD8GFP animals. Individual muscles are shown and color-coded as follows: levator muscles, turquoise; depressor muscles, blue; reductor muscles, pink; long tendon muscles, yellow. In the tibia ( $\boldsymbol{C}^{\prime \prime \prime}$ )', several sensory neuron cell bodies are also labeled by Vglut-Gal4 (indicated by the *). Co, Coxa; Tr, trochanter; Fe, femur; Ti, tibia; Ta, tarsus; trlm, trochanter levator muscle; trdm, trochanter depressor muscle; trrm, trochanter reductor muscle; fedm, femur depressor muscle; ferm, femur reductor muscle; Itm2; long tendon muscle 2; tilm, tibia levator muscle; tidm, tibia depressor muscle; tirm, tibia reductor muscle; Itm1, long tendon muscle 1; talm, tarsus levator muscle; tadm, tarsus depressor muscle; tarm, tarsus reductor muscle (Soler et al., 2004). D, CNS preparations from adult Vglut-Gal4; UAS-CD8GFP flies imaged for GFP fluorescence. $\boldsymbol{D}^{\prime}$ shows the thoracic and abdominal ganglia; $\boldsymbol{D}^{\prime \prime}$ shows a higher magnification of the T1 portion of the thoracic ganglia. The T1 neuromeres are outlined by the blue dotted circles. $\mathbf{D}^{\prime \prime}$ shows a lower-intensity version of the image in $\mathbf{D}^{\prime \prime}$ to better visualize the labeled cell bodies that lie immediately anterior and posterior to the neuromere (arrows). Some interneurons along the midline (indicated by the vertical blue dotted line in $\boldsymbol{D}^{\prime \prime}$ ) are also labeled by Vglut-Gal4.

$\mathrm{MeV}$ (MultiExperiment Viewer) version 4.2 (http://www.tm4.org/ mev.html/); Pearson's correlation and Euclidean distance methods were used for generating the sample tree (clustering motor neurons) and section tree (clustering neuromere sectors), respectively.

\section{Results}

Motor neurons in the legs and CNS

To study the development of the Drosophila leg motor neurons, we performed a clonal analysis using a modified version of mosaic analysis with a repressible cell marker (MARCM) method (Lee and Luo, 1999). We used the Vglut-Gal4 (also called
OK371-Gal4) driver to positively label clones (Mahr and Aberle, 2006). This Gal4 driver, which is inserted into the Vglut gene, is expressed in all neurons that use glutamate as a neurotransmitter, including all motor neurons (Jan and Jan, 1976; Mahr and Aberle, 2006). As can be seen in adult leg preparations in which Vglut-Gal4 was used to express a membrane-tagged version of green fluorescent protein (CD8GFP), motor neurons innervating all of the muscles in the coxa (co), trochanter (tr), femur (fe), and tibia (ti) were labeled by this driver (Fig. 1A). In addition, a subset of sensory neurons, whose cell bodies reside in the tibia 

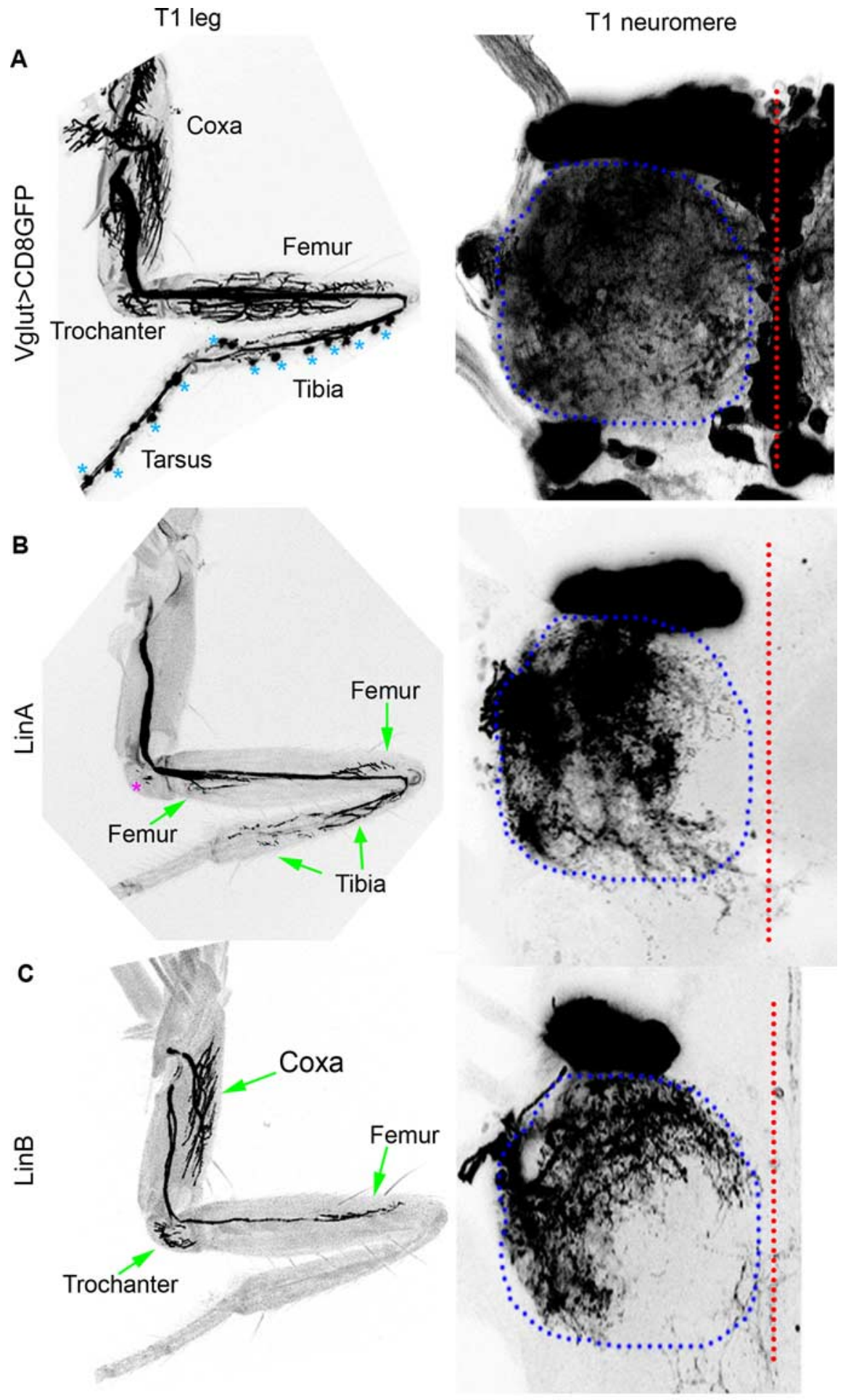

Figure 2. Leg motor neurons born from lineages A and B. A, Dissected leg (left) and T1 neuromere (right; outlined in blue) from a Vglut-Gal4; UAS-CD8GFP adult. Individual leg segments are labeled. The blue asterisks ${ }^{*}$ ) indicate the cell bodies of sensory neurons that are also labeled by this driver. The neuromere images in $\boldsymbol{A}-\boldsymbol{C}$ are projections of the entire Z-stack along the dorsoventral axis. $\boldsymbol{B}$, Dissected leg (left) and $\mathrm{T} 1$ neuromere (right; outlined in blue) from an animal containing a positively marked Lin A clone that labels axons in the femur and tibia (arrows). The purple asterisk indicates a non-Lin A motor neuron that was also labeled in this sample. C, Dissected leg (left) and T1 neuromere (right; outlined in blue) from an animal containing a positively marked Lin B clone, which labels axons in the coxa, trochanter, and femur (arrows).

and tarsal segments, were labeled by Vglut-Gal4. Except for the tarsus, each leg segment has a stereotyped set of multifibered muscles that are labeled by the MHC-tauGFP reporter gene (Fig. 1B) (Soler et al., 2004; Maqbool and Jagla, 2007). We used this reporter gene to identify each of the muscles innervated by the leg motor neurons (defined by Soler et al., 2004) (Fig. 1B,C). In the adult CNS, Vglut-Gal4 labeled groups of neurons in each thoracic hemisegment (Fig. $1 D$ ). In addition to motor neuron cell bodies, the dendritic arbors of these neurons were observed in densely packed neuropils in each thoracic hemisegment (Fig. 1D). We focused in this study on the motor neurons innervating the first thoracic (T1) legs. The axons of these motor neurons fasciculate and exit the CNS through a large nerve that extends into the ipsilateral leg.

Using these tools, we asked two fundamental questions about the organization and development of leg motor neurons. First, how many independent lineages are there, and how many motor neurons does each lineage generate? Second, does lineage, birth date, dendritic arbor pattern, or cell body position in the CNS correlate with targeting to individual leg segments and muscles? (For segment and muscle definitions, see Figure 1.)

The number of independent lineages revealed by embryonic MARCM clones Drosophila NBs are born during embryogenesis and undergo two waves of neurogenesis, one during embryogenesis and one during larval development (Truman and Bate, 1988; Prokop and Technau, 1991; Maurange and Gould, 2005). During the first, embryonic wave of NB divisions, the majority of the embryonically born neurons are dedicated to larval motor and sensory functions and die during metamorphosis. To determine how many independent NB lineages give rise to the leg motor neurons, we induced positively labeled MARCM clones during embryogenesis and analyzed them in the adult. Because these clones were generated infrequently and early in development, entire NB lineages were labeled. These data revealed that the leg motor neurons are derived from at least 11 independent lineages (Table 1). Strikingly, two of these lineages, Lin A and Lin B, give rise to the majority of the leg motor neurons (Fig. 2, Table 1). Embryonically induced clones of Lin A innervated the muscles of the femur and tibia but did not include any motor neurons that targeted the coxa or trochanter. Moreover, the tibia is only targeted by Lin A-derived motor neurons. Thus, Lin A motor neurons generally target distal, but not proximal, leg segments.

The second major lineage defined by these experiments is Lin $\mathrm{B}$, which gives rise to seven leg motor neurons (Fig. 2, Table 1). In contrast to Lin A, Lin B motor neurons target the three most proximal leg segments, the coxa, 
trochanter, and femur, but does not generate any motor neurons that target the tibia. Thus, Lin B motor neurons generally target proximal, rather than distal, leg segments.

Embryonically induced MARCM clones revealed that another 12 Vglut-Gal4 $^{+}$leg motor neurons are generated from nine additional lineages, Lin $\mathrm{C}$ to Lin $\mathrm{K}$ (Table 1). These 12 motor neurons target the coxa (six), the trochanter (one), and the femur (five), but not the tibia (Table 1). In contrast to Lin A and Lin B, these lineages give rise to only one or two Vglut-Gal4-expressing leg motor neurons. Lin $\mathrm{E}$ is distinctive because, in addition to generating a single motor neuron targeting the coxa, it also gives rise to $\sim 25 \mathrm{Vglut-Gal4-expressing}$ interneurons. Five of these lineages $(\mathrm{C}$ to $\mathrm{G}$ ) were labeled frequently, by both embryonic and postembryonic heat shocks. In contrast, four of these lineages, Lin $\mathrm{H}$ to Lin $\mathrm{K}$, were labeled infrequently and only by embryonic heat shocks (Table 1). These findings suggest that these motor neurons, which target the coxa (one) and femur (five), are born during embryogenesis and persist to the adult stage in which they contribute to the adult leg nervous system.

In addition to the 47 motor neurons generated by Lin A to Lin $\mathrm{K}$, we identified six additional Vglut-Gal4 ${ }^{+}$motor neurons that were not labeled by inducing clones during embryogenesis (Table 1). Two of these motor neurons, one targeting the coxa and one the trochanter, were not labeled in any of our MARCM experiments but could be identified in Vglut-Gal4; UAS-CD8GFP adult flies. The other four motor neurons, which we tentatively label MN V to MN Y, all target the femur and were only labeled by postembryonic clones. Because these motor neurons were not labeled by embryonic MARCM experiments, we cannot exclude the possibility that they belong to one of the lineages defined above. Alternatively, they may be derived from independent lineages that are difficult to label during embryogenesis. Nevertheless, together with the 47 embryonically labeled motor neurons, these results reveal 53 motor neurons that innervate the T1 leg.

\section{Single-cell analysis of leg motor neurons}

To examine individual motor neurons derived from these lineages, we induced positively labeled MARCM clones postembryonically. In addition to occasionally labeling subsets of Lin A and Lin B (Table 1 and data not shown), these clones most typically labeled individual leg motor neurons, enabling us to characterize axon targeting, cell body position, and dendritic arbors for individual neurons in the adult CNS. In addition, by varying the time of clone induction (using $2 \mathrm{~h}$ intervals), we measured the birth date for each motor neuron. Individual motor neurons were characterized by (1) which leg segment they targeted, (2) which muscle they targeted, (3) their axonal branching pattern, and (4) their dendritic arbor pattern in the CNS (see Materials and Methods). Importantly, many independent examples of the same labeled motor neuron show that they each have stereotyped dendritic and axon arbors that are recognizable from animal to animal (Fig. 3A,B) (supplemental Fig. 1, available at www. jneurosci.org as supplemental material).

\section{Lineage A}

Single-cell labeling experiments reveal that Lin A generates 15 motor neurons that target the tibia and 13 motor neurons that target the femur. Based on the latest time when the full set of Lin A motor neurons was labeled, they are born starting at $\sim 50 \mathrm{~h} \mathrm{AEL}$ (Table 1). Based on this timing, and the number of motor neurons generated, Lin A is likely to be the same as lineage 15, characterized previously in the CNS of third-instar larvae (Truman et al., 2004). Representative single cell clones for 27 of these 28 motor neurons are shown in supplemental Figures 2 (tibia) and 3 (femur) (available at www.jneurosci.org as supplemental material), and a subset of these are shown in Figure 4. For example, the long tendon muscle in the tibia (ltm1) is targeted by nine Lin A motor neurons. Interestingly, these axons terminate at distinct positions along this muscle, suggesting that it is compartmentalized (Fig. 4).

\section{Lineage B}

The seven leg motor neurons derived from Lin B target the coxa (four), the trochanter (two), and the femur (one) (Fig. 5). Our postembryonic MARCM data suggest that the Lin B neuroblast begins to generate its leg motor neuron progeny at $\sim 54 \mathrm{~h} \mathrm{AEL}$, because all seven neurons can be labeled up to this time of development (Table 1). Single-cell clones for all seven of these motor neurons are shown in Figure 5. As with Lin A motor neurons, the dendritic patterns of the Lin B motor neurons each have a stereotyped pattern within the T1 neuromere (Fig. 5).

\section{Minor lineages}

In addition to Lin A and Lin B, the MARCM analysis suggests that an additional nine NB lineages ( Lin $\mathrm{C}$ to Lin $\mathrm{K}$ ) give rise to 12 leg motor neurons (Table 1) (supplemental Fig. 4, available at www.jneurosci.org as supplemental material). As with Lin A- and Lin B-derived motor neurons, the dendritic patterns and axonal projections of the motor neurons derived from Lin $\mathrm{C}$ to Lin $\mathrm{K}$ were consistent from animal to animal (supplemental Fig. 4, available at www.jneurosci.org as supplemental material).

In summary, we have defined 11 independent NB lineages that give rise to $\sim 50$ motor neurons that target the T1 leg. Apart from the finding that Lin A motor neurons only target the two more distal leg segments and Lin B motor neurons target the three more proximal leg segments, there are no PD targeting restrictions for these lineages. Below, we analyze whether muscle and/or PD targeting correlates with other characteristics of these motor neurons, such as birth date, cell body position, or dendritic pattern.

\section{Cell body position}

Fifty of the 53 T1 leg motor neurons have their cell bodies clustered together anterior to the T1 neuropil in the CNS (Fig. 1D). Within the level of resolution of our experiments, we cannot detect any organization of these cell bodies within this anterior group of motor neurons. The remaining three motor neurons have cell bodies that lie posterior to the neuropil. Although all three of these neurons target the coxa, they are derived from three separate lineages (Table 1). In addition to these three motor neurons, seven other motor neurons, with anteriorly positioned cell bodies, target the coxa. Thus, other than those cells positioned posterior to the neuropil, cell body position is a poor predictor of motor neuron targeting in the Drosophila leg.

\section{Birth date}

The existence of NB lineages suggests that individual motor neurons may be born at highly restricted and characteristic times during development. To test this, we plotted the birth dates for each of the 44 motor neurons for which we have been able to label as single cells in postembryonic MARCM experiments (Fig. 6). Strikingly, each motor neuron, defined by its muscle target and dendritic pattern, is born within a stereotyped and narrow window of development (Fig. 6). For Lin A, which gives rise to 28 motor neurons during a $\sim 40 \mathrm{~h}$ time window, a clear temporal birth order was observed (Fig. $6 A$ ). 

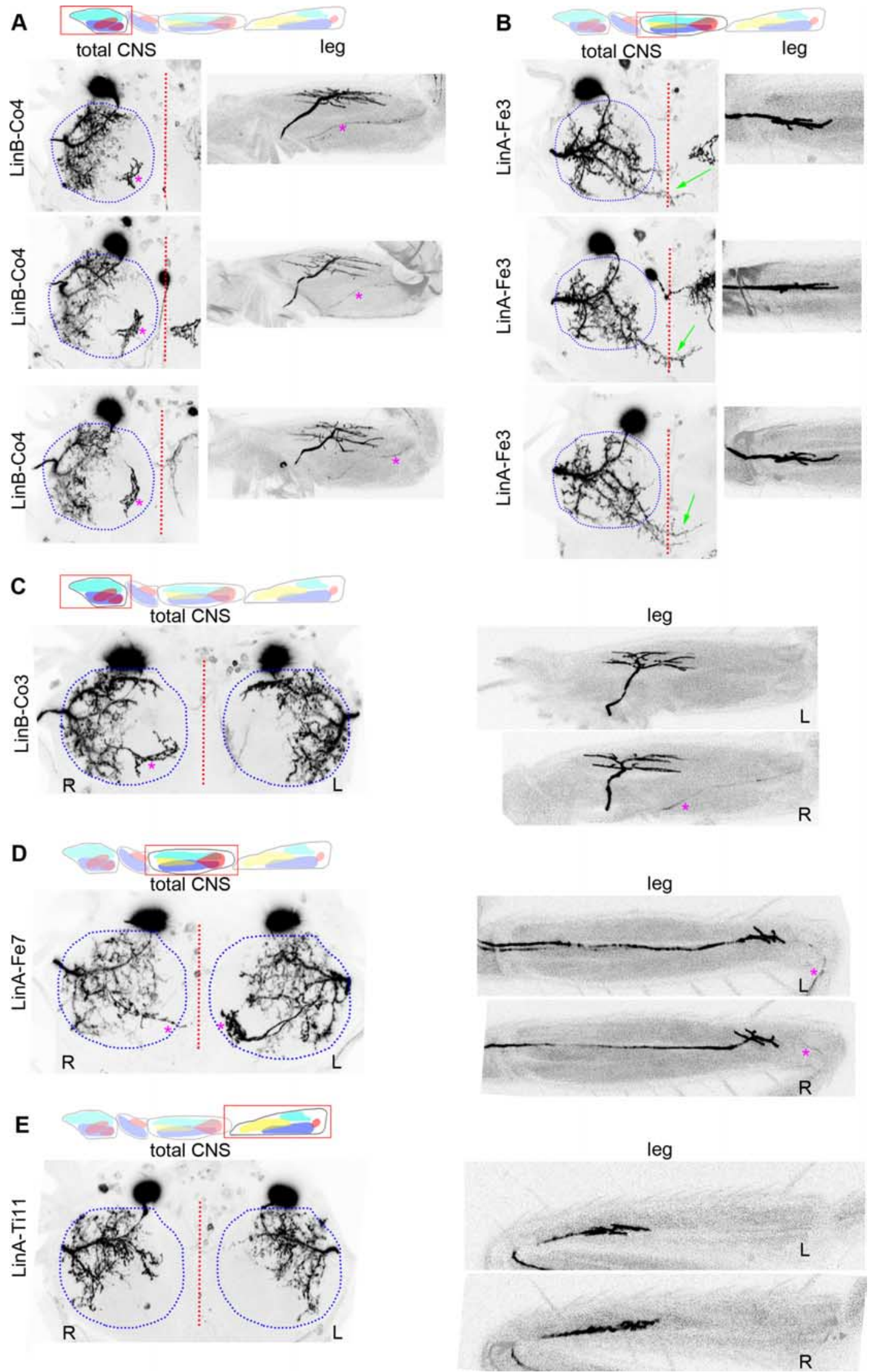

Figure3. Reproducibility of dendrite and axon arbors. $\boldsymbol{A}, \boldsymbol{B}$, Three examples of the same Lin B-derived motor neuron (C04; $\boldsymbol{A}$ ) and the same Lin A-derived motorneuron (Fe3; $\boldsymbol{B})$ showing very similar dendritic (leftimages) and axon (rightimages) arbors. Theschematics abovetheimagesindicatetheimagedlegsegment (red boxes). Thepurpleasterisksindicatesensoryneurons that werealso labeled in theseexperiments. Theneuromereimages are projections of the entire dorsoventral axisZ-stack. A subset of Lin A-Fe3 dendrites extend across the midline ( $\boldsymbol{B}$, arrows). The midlines of the CNSs areindicated by the red dotted lines. $\boldsymbol{C}-\boldsymbol{E}$, Three examples of samples that had theidentical motorneuron labeled on the leftand rightsides of the sameanimal. In each case, boththeleft $(L)$ and right $(R)$ neuromeres (leftpanels) andlegs (right panels) areshown. Theschematicsabovetheimages indicatethe imaged leg segment (red boxes). C, Lin B-C03; D, Lin A-Fe7; $\boldsymbol{E}$, Lin A-Ti11. Purple asterisks indicate sensory neurons that were also labeled in these images. The midlines of the CNSs are indicated by the red dotted lines. 
Lin A tibia
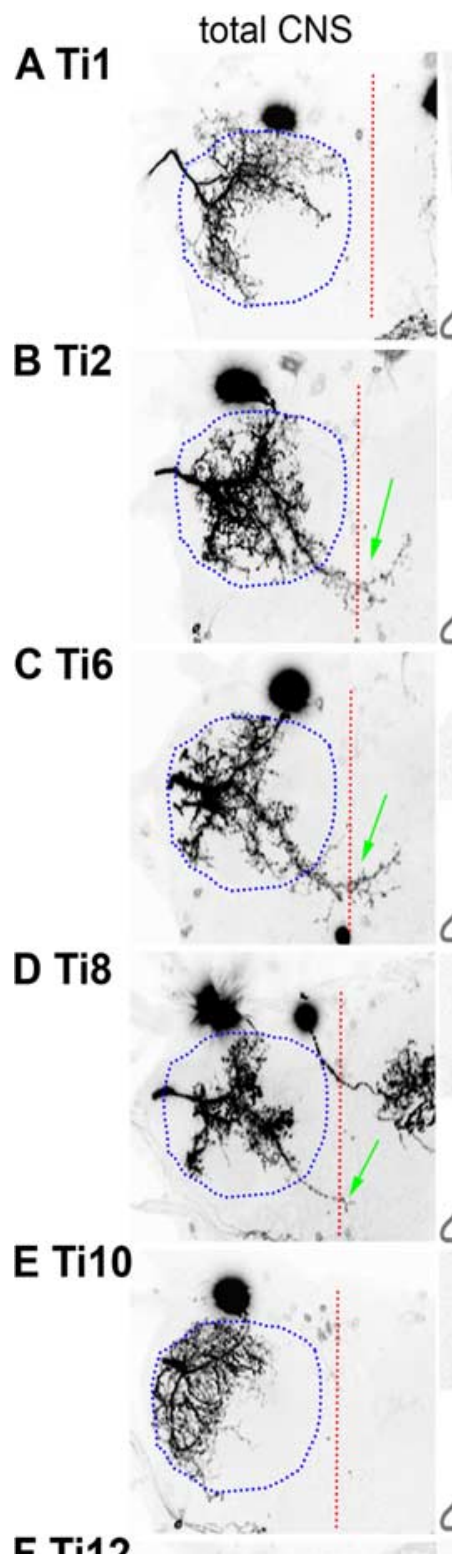

F Ti12

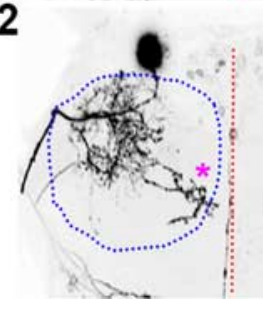

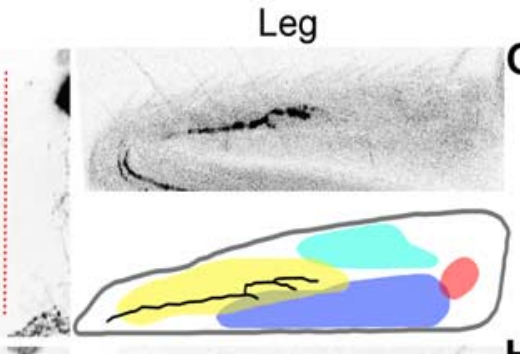
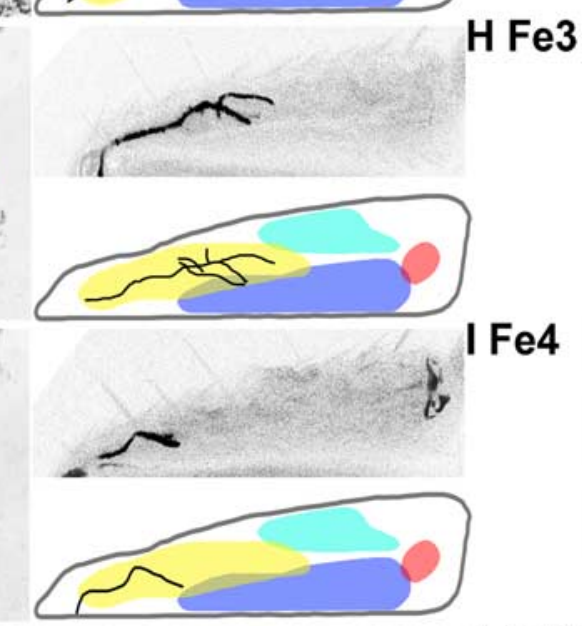

J Fe8
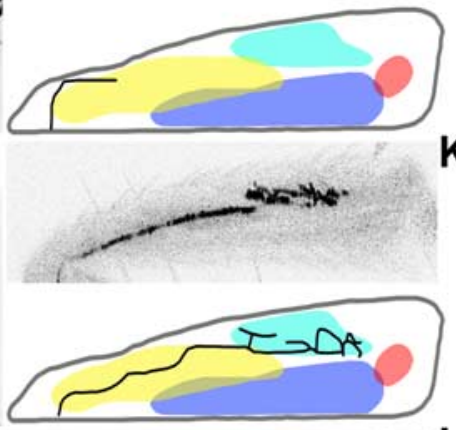

K Fe9

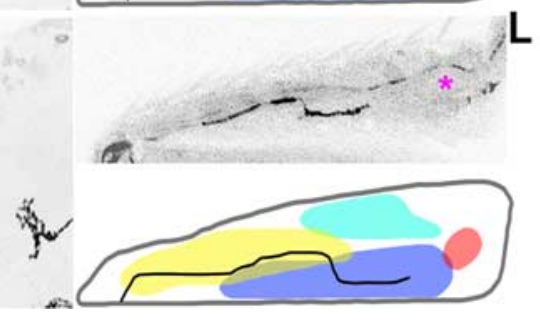

$\mathrm{HFe} 3$

Fe10

total CNS
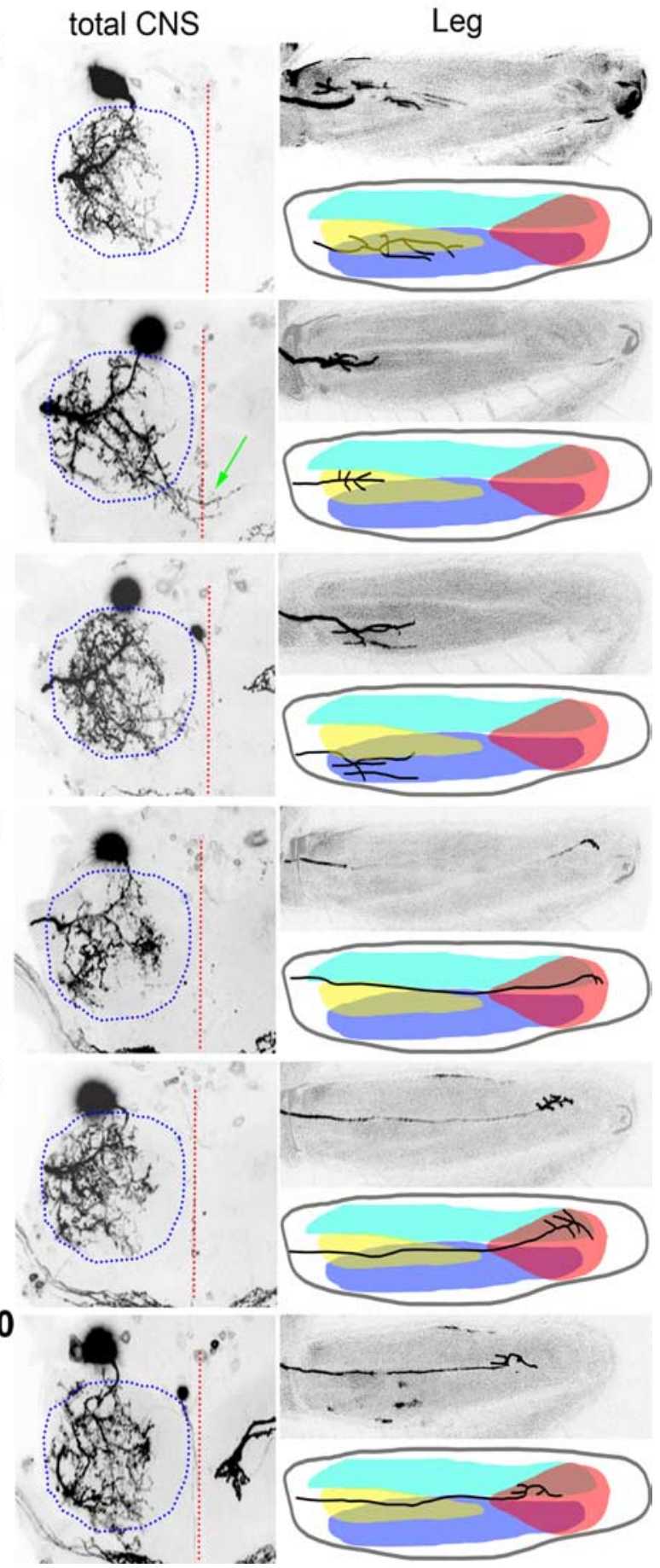

Figure 4. Representative Lineage A motor neurons. $\boldsymbol{A}-\boldsymbol{F}$, Representative Lin A motor neurons that target the tibia. In all cases, the dendrites in the neuromere (a compression of the entire dorsoventral stack), the axon in the leg, and a schematic of the leg segment and axon are shown. The midlines of the CNSs are shown by the red dotted lines. Ti1 $(\boldsymbol{A})$ and Ti2 ( $\boldsymbol{B})$ target the distal part of the long tendon muscle 1 (Itm1; schematized in yellow), and Ti6 (C) and Ti8 (D) target the proximal part of the same muscle. Note that Ti2, Ti6, and Ti8, which all target Itm1, all have midline-crossing dendrites (arrows). Ti10 and Ti12 target the tarsal levator (talm) and tarsal depressor (tadm), respectively. Red asterisks indicate sensory neurons. $\mathbf{G}-\mathbf{L}$, Representative Lin A motor neurons that target the femur. Note that Fe3 $(\boldsymbol{H})$, which targets Itm2, has midline-crossing dendrites. Fe1 and Fe4 target the tibia depressor (tidm), and Fe8, Fe9, and Fe10 target the tibia reductor (tirm). For examples of all 27 of the 28 Lin A motor neurons that have been labeled as single cells, see supplemental Figures 2 and 3 (available at www.jneurosci.org as supplemental material).

That each motor neuron has a stereotyped birth date is also illustrated by analyzing animals with more than one labeled motor neuron. Although the vast majority of the samples we analyzed (408 of 428) had only a single labeled leg motor neuron, 17 samples had two labeled leg motor neurons (one on each side) and three had three labeled motor neurons. For each of these 20 samples, the birth dates (as measured using all available data) of the neurons labeled in the same animal were within $2 \mathrm{~h}$ of each other (supplemental Fig. 5, available at www.jneurosci.org as supplemental material). Moreover, of the 20 samples that had more than one labeled motor neuron, seven had the identical motor neuron labeled on both sides of the animal (Fig. 3C-E) 
Lin B

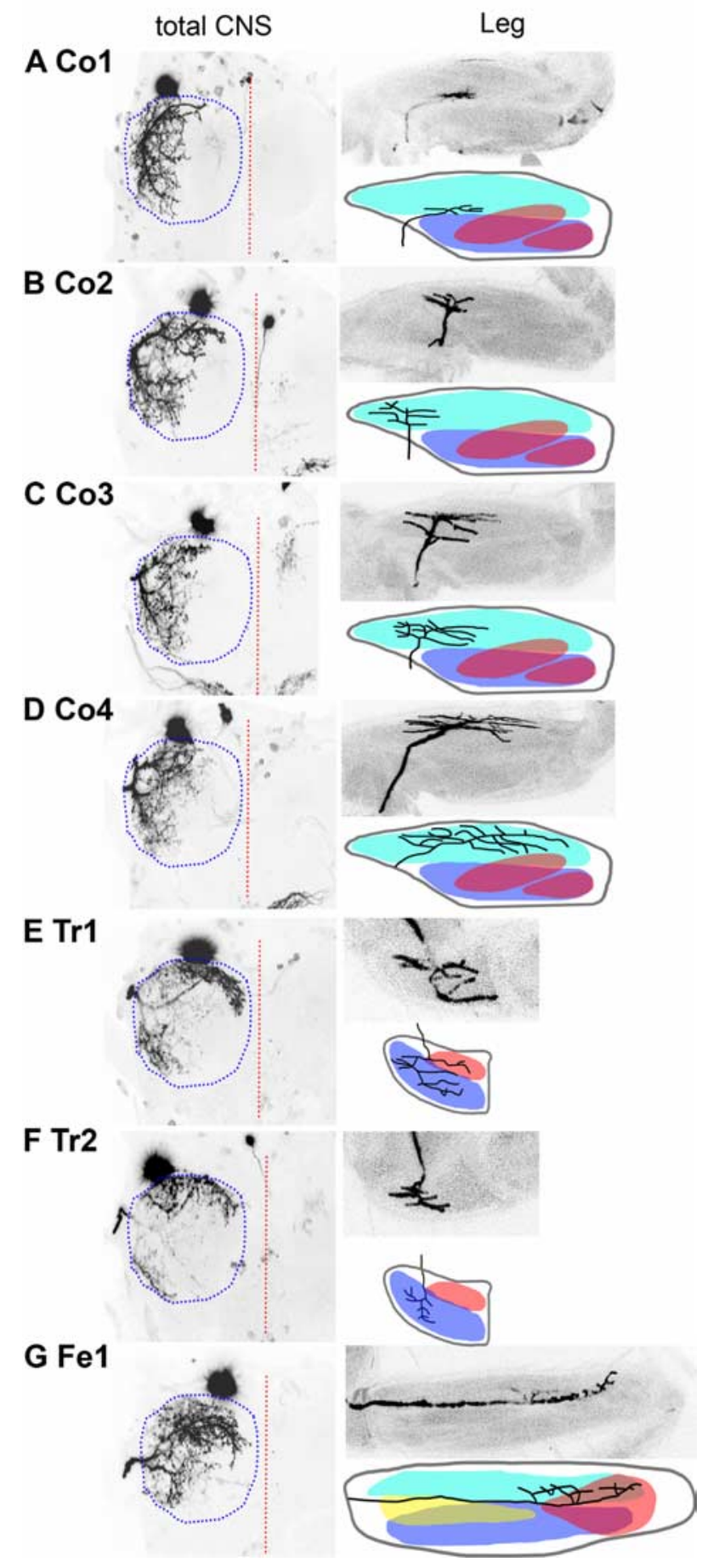

Figure 5. Lineage $B$ motor neurons. $A-G$, Representative examples of all seven $L$ in $B$ motor neurons. $\boldsymbol{A}-\boldsymbol{D}$ (C01 to $\mathrm{C} 04$ ) project to the coxa, $\boldsymbol{E}$ and $\boldsymbol{F}$ ( $\mathrm{Tr} 1, \mathrm{Tr} 2)$ project to the trochanter, and $\mathbf{G}(\mathrm{Fe} 1)$ projects to the femur. Images and labeling are the same as for Figure 4. Four motor neurons (Lin B-C01-4; $A-D)$ target the trochanter levator muscle in the coxa $(\operatorname{trl} I m)$. In the trochanter, Lin B-Tr1 targets the femur reductor muscle (ferm; $\boldsymbol{E}$ ) and the femur depressor muscle (fedm). Lin B-Tr2 targets the femur depressor muscle (fedm; $\boldsymbol{F}$ ). In the femur, a single Lin B motor neuron, Fe1 $(\boldsymbol{G})$, targets both the tibia depressor muscle (tidm) and the tibia reductor muscle (tirm). Midlines in the CNS images are indicated by the red dotted lines.

(supplemental Fig. 5, available at www.jneurosci.org as supplemental material). These observations argue that the left and right versions of the same lineages within an individual animal are dividing with remarkable synchrony.
We next asked whether there was a correlation between birth order and target position along the PD axis of the leg. For Lin A, early-born neurons target both the femur and tibia; similarly, late-born neurons also target both of these leg segments. Thus, for this lineage, there is no correlation between birth date and targeting a particular leg segment. However, there is a correlation between birth order and PD targeting within individual leg segments. The first 15 motor neurons born from Lin A all target muscles in the proximal tibia or proximal femur, whereas 10 of the 12 last born Lin A motor neurons target distally positioned muscles within these segments (Fig. 6A). Another way to describe this correlation is to say that 11 of the first 15 born Lin A neurons target the long tendon muscles (ltm 1 and $\operatorname{ltm} 2)$, which are positioned proximally in both the femur and tibia, whereas 8 of the 12 late born neurons target reductor muscles (tirm and tarm), which are positioned distally within these two leg segments. For Lin B, the three last motor neurons to be born all target the coxa levator muscle (Fig. 6B). Otherwise, no correlations with $\mathrm{PD}$ position or muscle type were observed for Lin B motor neurons.

As noted above, Lin A generates nine motor neurons that target the long tendon muscle in the tibia (Fig. $4 A-D$ ) (supplemental Fig. $2 A-I$, available at www.jneurosci.org as supplemental material). Interestingly, axon targeting within this muscle correlates with motor neuron birth order: the first two neurons that are born target distal positions in the muscle, whereas subsequently born motor neurons target more proximal positions (Fig. 4A-D) (supplemental Fig. 2A-I, available at www.jneurosci.org as supplemental material).

\section{Dendritic arbors}

Inspection of the dendrites of individually labeled leg motor neurons suggests that neurons that project to a particular leg segment have similar dendritic architectures. For example, the dendrites of the four coxa-targeting neurons derived from Lin B appear to have a characteristic shape and position within the neuropil that are distinct from those of the trochanter- and femur-targeting neurons from the same lineage (Fig. 5). To examine this in more detail, we quantified the contribution of the dendrites of each motor neuron to one of eight sectors (D1 to D4 and V1 to V4) within the neuropil using quantitative three-dimensional confocal imaging of GFP-labeled neurons (Fig. 7A) (for details, see Materials and Methods). Performing this analysis on independent samples of the same labeled motor neuron shows that they have reproducible and distinct architectures within the neuropil (supplemental Fig. 1, available at www.jneurosci.org as supplemental material).

We performed the eight-sector analysis on the dendritic arbors for representative examples of all 47 motor neurons that were individually labeled in our MARCM experiments. Using these data, which provide a measurement of the spatial organization of each dendritic arbor in the neuropil, we asked whether these patterns correlate with other motor neuron characteristics, such as birth date or muscle targeting. Although no correlations between dendritic pattern and birth date were observed, several other correlations were apparent. For example, of the 47 leg motor neurons analyzed, only eight have dendrites that cross the midline in the CNS, contributing to a distinct dendritic pattern (Fig. $7 B$ ). Interestingly, all eight of these neurons target the long tendon muscles (ltm 1 and ltm2). Because ltm 1 is in the tibia and $\operatorname{ltm} 2$ is in the femur, this midline-crossing property is independent of leg segment. A second striking example of a shared dendritic organization is seen for the three motor neurons that target the trochanter (Fig. 7C). For all three of these motor neurons, the 


\section{A $\operatorname{Lin} A$}

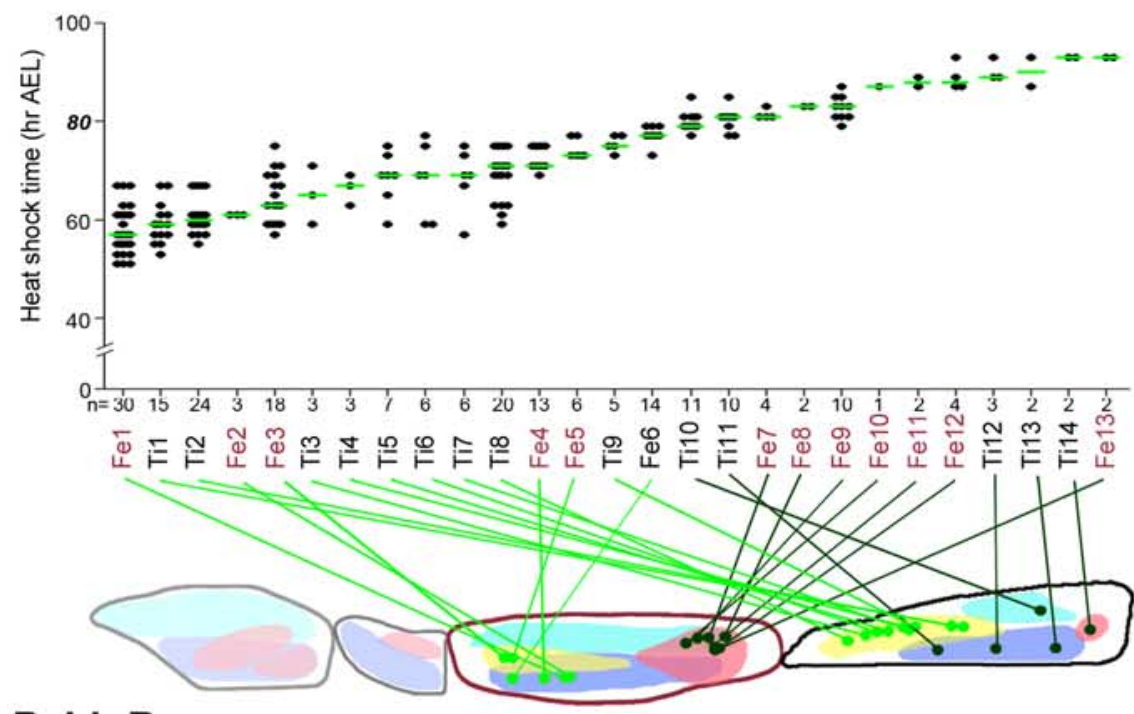

\section{B LinB}

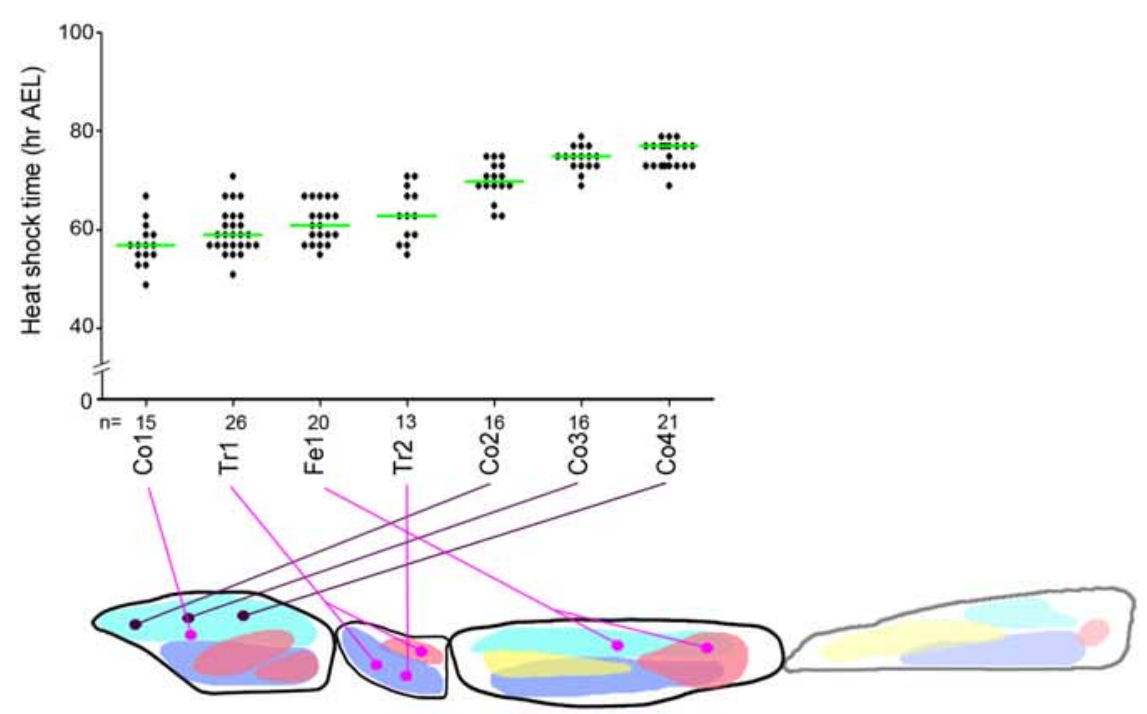

C LinC-F, MN V-Y

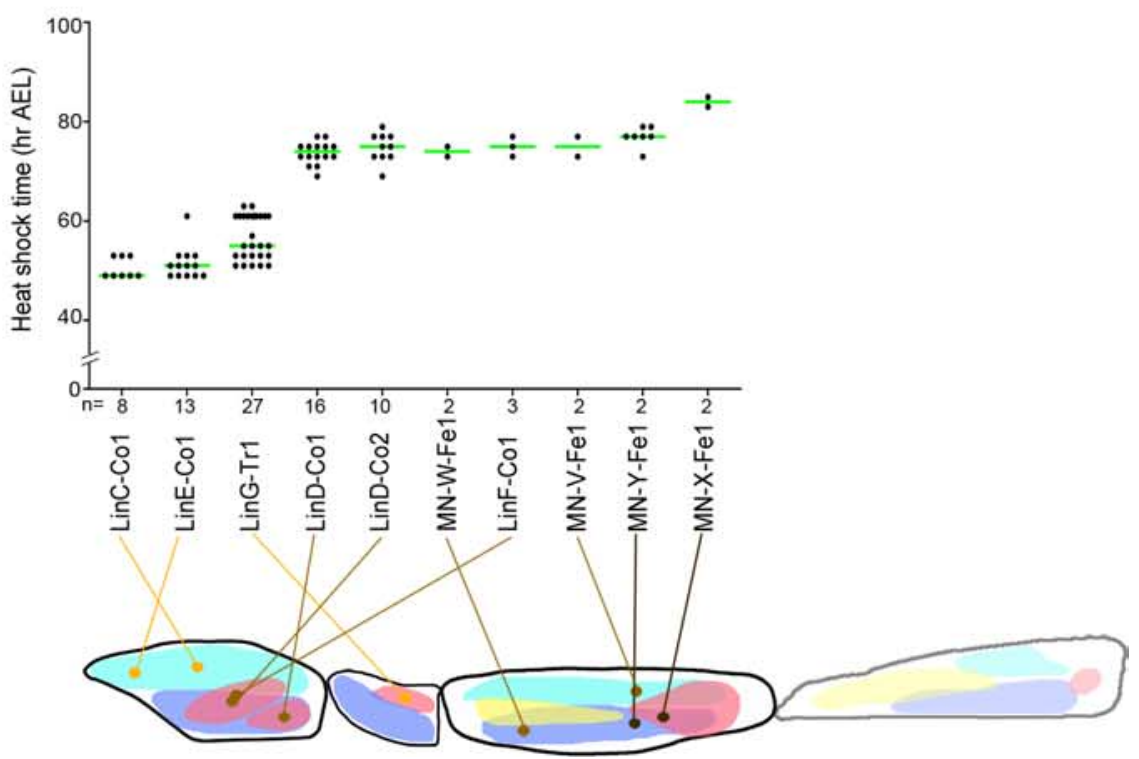

dendritic arbors are biased toward the anterior and ventral side of the neuromere, and there is a noticeable absence from the posterior and medial regions of the neuromere (Fig. 7C). Thus, these and other (supplemental Fig. 6, available at www. jneurosci.org as supplemental material) examples suggest that there is a correlation between dendritic arbor position in the CNS and axon targeting in the leg.

Based on these initial observations, we next asked whether the dendritic organization of leg motor neurons correlates with targeting. To assess this issue in an unbiased manner, we generated dendrograms using the eight-sector data for all 47 motor neurons (Fig. 7D). Strikingly, this analysis resulted in the clustering of at least four functionally related sets of motor neurons. Two of these sets were the trochantertargeting neurons and the midlinecrossing neurons described above, demonstrating that this approach is able to identify similar dendritic patterns. In addition, two other sets of motor neurons clustered together: those that target the coxa and those that target reductor (as opposed to levator or depressor) muscles (Fig. 7D) (supplemental Fig. 6E, G, available at www.jneurosci.org as supplemental material). Within each of these four groups of motor neurons, we also found that different combinations of sectors correlated with each other (Fig. $7 E$ ). For example, the dendrites of the coxa-targeting group of motor neurons are underrepresented in sectors D2, D4, and V4, but these same sectors are not correlated with each other in the midline-crossing group of motor neurons. Instead, the dendritic arbors of the midline-crossing group show correlations between V1, V2, and V3 (in which they are underrepresented) and between D1, D2, and D3 (in which they are overrepresented) (Fig. 7E). Together,

\footnotetext{
$\leftarrow$

Figure 6. Relationships between birth date and muscle targeting. $A-C$, The birth dates ( $\mathrm{h} \mathrm{AEL}$ ) and muscle targets are plotted for 27 Lin A $(\boldsymbol{A}), 7$ Lin B $(\boldsymbol{B})$, or 10 other $(\boldsymbol{C})$ motor neurons that have been individually labeled. Each black dot represents a unique single-cell clone for that motor neuron, and the green bars represent the median birth date. Motor neurons are ordered along the $x$-axis according to median birth date. The number of samples for each individually labeled motor neuron immediately follows its name. For neurons that were only rarely labeled (e.g., Lin A Fe10), birth dates are tentative. For $\operatorname{Lin} A(\boldsymbol{A})$, motor neuron names are red or black, depending on if they project to the femur or tibia, respectively. Also for Lin $A(A)$, the lines going to the muscles are colored light or dark green for motor neurons born during the first or second halves, respectively, of the time window when motor neurons are being generated by this lineage.
} 
A
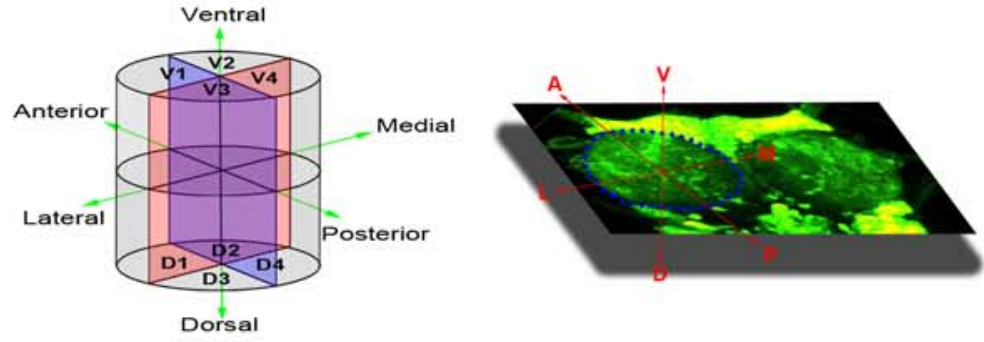

B Midline crossing - 8 cells

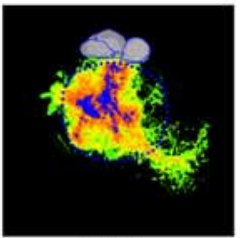

total

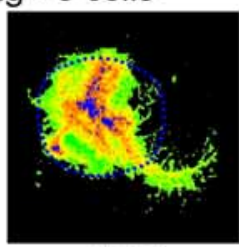

dorsal

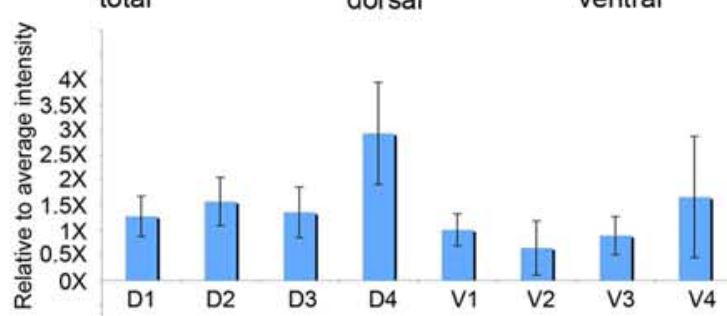

C Trochanter-targeting -3 cells
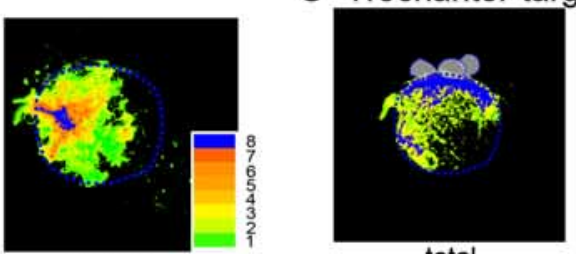

total

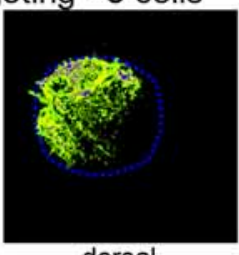

dorsal

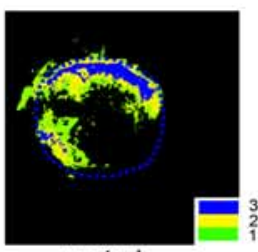

ventral

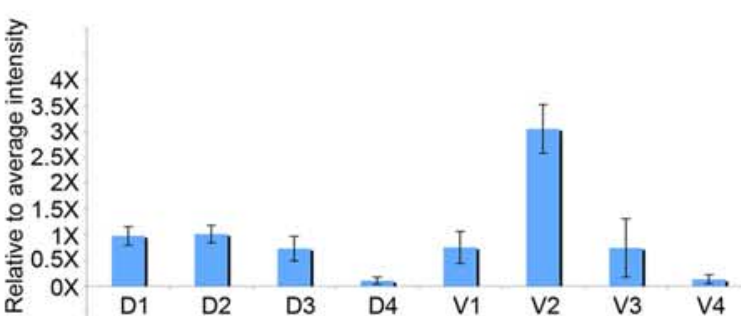

D

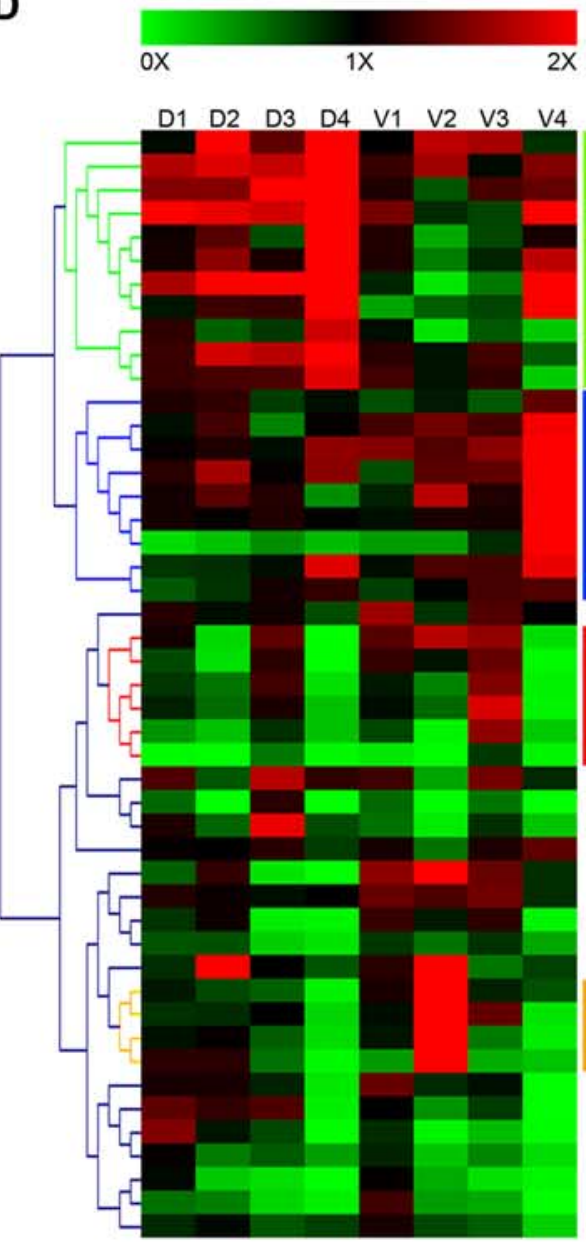

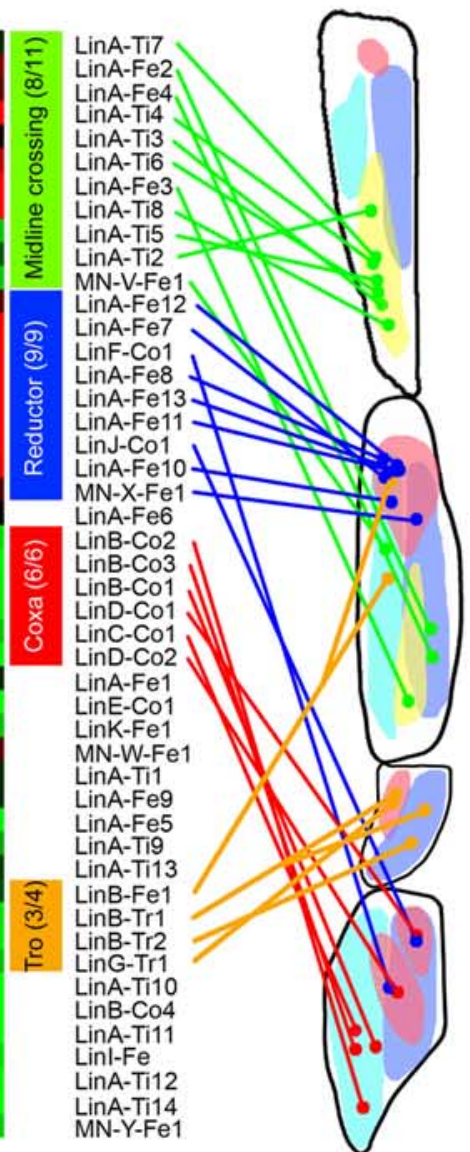

E
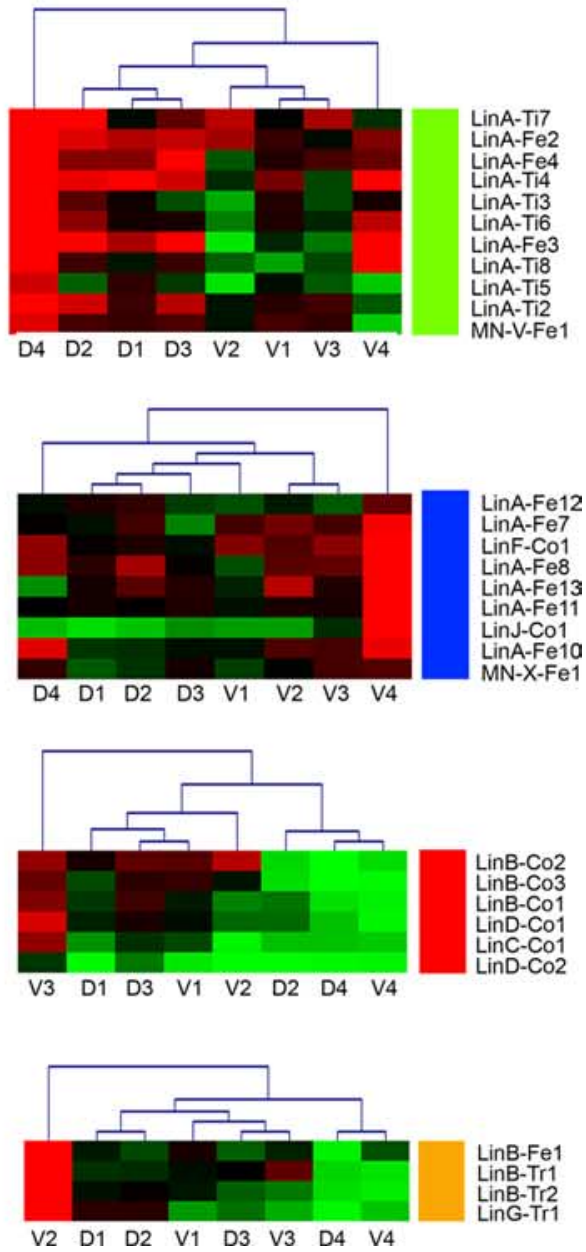
these observations reinforce the conclusion that neurons with shared targets often have similar dendritic architectures, whereas those that target different muscles or leg segments have distinct dendritic patterns.

\section{Discussion}

Fruit flies walk using multi-jointed legs that are controlled by a stereotyped set of muscles in each leg segment. The contraction of muscles controlling the position and angle of each leg joint, between leg joints within a leg, and between legs on the right and left sides of the animal, must be highly coordinated. Each of these muscles is innervated by motor neurons that have cell bodies in the CNS. Thus, the anatomy and function of the adult fly motor system shares many aspects with vertebrate motor systems. To assess the degree these systems share a similar developmental logic, we characterized the development of the motor neurons that innervate the adult Drosophila leg. We identify 53 neurons, derived from 11 independent NBs, that innervate the T1 leg. Two of these lineages give rise to 35 of these 53 motor neurons. By characterizing individually labeled motor neurons, we determined the birth dates, muscle targets, and dendritic arbors for most of these motor neurons. These results show that, although each motor neuron is born from a specific lineage, and at a specific time during development, individual lineages give rise to motor neurons that target multiple leg segments and multiple muscles within these leg segments.

Birth date and lineage in postembryonic neural development Accurate motor neuron development in the fly requires that axons target the correct muscles along the PD axis of the leg. This axis has several levels of refinement. The first level is the global PD axis of the leg. We find that Lin A only generates motor neurons that target the two more distal leg segments, the tibia and the femur. In addition, Lin A is the only lineage that produces motor neurons that target the tibia. In contrast, the seven Lin B motor

\footnotetext{
$\leftarrow$

Figure 7. Relationships between dendritic pattern and muscle targeting. $\boldsymbol{A}$, Scheme for collecting eight sector data for dendrite occupancy in the $\mathrm{T} 1$ neuromere. The image on the right is a projection of $\sim 40$ confocal slices along the dorsoventral axis of the T1 neuromere. The cylinder on the left schematizes the three-dimensional $\mathrm{T} 1$ neuromere. Three axes are labeled: lateromedial, anteroposterior, and dorsoventral. The cylinder was divided into eight sectors (D1, D2, D3, D4, V1, V2, V3, V4) as indicated. For each labeled dendrite, the dorsal and ventral halves consisted of $\sim 20$ confocal sections. $B, C$, Examples of unique dendritic architectures within the T1 neuromere. The three images show heat maps quantifying the extent of overlap of the dendrites for the midline-crossing motor neurons $(\boldsymbol{B})$ and the trochanter-targeting motor neurons (C). The scales shown on the bottom right of each panel range from $100 \%$ overlap (blue) to no overlap (green; only a single dendrite is present). Heat maps were generated using confocal projections for all (total), dorsal, or ventral sections as indicated. The bar graphs below the images show the amount of dendrite representation for each set of neurons for all eight sectors; dendrite representations are expressed relative to the average for all motor neurons. The error bars are SDs. The dendrites of the midline-crossing neurons $(\boldsymbol{B})$ are overrepresented in sector D4, whereas those of the trochanter-targeting neurons $(\boldsymbol{C})$ are underrepresented in V4 and D4 and overrepresented in V2. Supplemental Figure 6 (available at www.jneurosci.org as supplemental material) presents analyses of dendrite organization for other related groups of motor neurons. $\boldsymbol{D}$, Dendrogram and heat map analysis of the eight sector data. For each motor neuron, each sector was given a dendritic representation score relative to the average representation for all 47 motor neurons. The figure color codes these scores, ranging from $\leq 0.1 \times$ relative to the average (green) to $\geq 2 \times$ relative to the average (red). Black is equivalent to the average $(1 \times)$. Motor neurons were clustered according to the similarities in their eight sector data using MeV (http://www.tm4.org/mev.html). Groups of motor neurons that cluster together are shown on the right, and their muscle targets in the legs are schematized. $\boldsymbol{E}$, For each of the four sets of motor neurons that clustered together in $(\boldsymbol{D})$, the columns (sectors) were clustered according to their similarities. Different sectors group together in the different sets of motor neurons, suggesting that each set of motor neurons has a unique dendritic organization.
}

neurons target all leg segments except the tibia. Thus, there is a PD bias built into these lineages.

A second level of refinement within the PD axis is targeting the correct muscle in individual leg segments. Among the Lin A-derived motor neurons, we observe a PD bias within the tibia and within the femur that correlates with birth date: the first half of the motor neurons born from Lin A have a strong bias for targeting proximal positions in these segments, whereas the laterborn half of the motor neurons target distal muscles in these segments.

Third, for muscles that are targeted by multiple motor neurons (e.g., ltm 1 in the tibia), we find that more distal projecting motor neurons are born before those that target more proximal positions in the same muscle. The differential targeting of axons to unique positions within the same muscle suggests the existence of high-resolution topographic maps that match specific motor neurons to specific muscle compartments, as has been observed in mouse skeletal muscles (Laskowski and Sanes, 1987; Feng et al., 2000).

Most of the leg motor neurons are born within a narrow window of development. The NB that gives rise to Lin A, for example, switches into a phase that is dedicated to generating leg motor neurons at $\sim 50 \mathrm{~h} \mathrm{AEL}$. At that time, this NB begins to produce its 28 motor neurons for the next $\sim 40 \mathrm{~h}$. Presumably, this NB gives rise to nonmotor neuron progeny before this time and possibly after it completes this motor neuron generating phase. This scenario shares some similarities with the lineages that give rise to postembryonic neurons in the fly brain. For example, the entire mushroom body of Drosophila, the portion of the fly brain used in olfactory learning and memory, is derived from only four NBs that each give rise to one of four nearly identical anatomical units (Lee et al., 1999; Ito and Awasaki, 2008). Interestingly, there is a temporal switch in the types of neurons that these NBs generate at specific times of development. Thus, like Lin A, mushroom body NBs switch the type of neuron they generate at specific times. However, unlike the leg motor neuron NBs, those that generate the mushroom body are dedicated to forming this brain structure. In contrast, we find that functionally related leg motor neurons, for example those that target a specific leg segment, muscle, or muscle type, are often derived from several different NB lineages. This logic is reminiscent of that used to generate olfactory projection neurons in the fly, in which three neuroblasts each give rise to different numbers and types of projection neurons (Jefferis et al., 2001).

The temporal control of NB identity in Drosophila is directed by transcription factors that are sequentially expressed as NBs age (Pearson and Doe, 2004; Brody and Odenwald, 2005; Doe, 2006; Jacob et al., 2008; Maurange et al., 2008). During embryogenesis, progeny postmitotic neurons inherit the transcription factor expressed in the NB at the time it was born. This temporal information works in combination with positional information that makes each NB unique, providing progeny neurons their individual identities. Although the specific factors are not yet known, a similar transcription factor code may exist for leg motor neurons. Two of the temporal control genes that are used during Drosophila embryogenesis, seven-up (svp) and castor (cas), are also important for controlling postembryonic neural fates (Maurange et al., 2008). Interestingly, some NBs switch from expressing cas to $s v p$ at $\sim 50 \mathrm{~h} \mathrm{AEL}$, similar to the time that the leg NBs begin to generate their leg motor neuron progeny. It will be interesting to determine whether this or other changes in transcription factors are responsible for initiating the production of leg motor neurons in the lineages defined here. 


\section{Lineage versus position}

Our results demonstrate that adult motor neurons in the fly come from identifiable lineages that give rise to stereotyped progeny with defined birth dates. Importantly, however, of the 11 lineages that give rise to leg motor neurons in the fly, only one of these, Lin A, appears to be dedicated to producing these neurons. Even this restriction only occurs during the $\sim 50$ to $\sim 90 \mathrm{~h}$ AEL time window. Although most of the progeny produced by the other lineages were not marked in our experiments (except for Lin E, which generates $\sim 25 \mathrm{Vglut}-\mathrm{Gal}_{4}{ }^{+}$interneurons), it is likely that these lineages also produce nonmotor neuron progeny (Truman et al., 2004). Thus, although seemingly invariant lineages are used in the fly, the closest relatives for many leg motor neurons are not other leg motor neurons. This conclusion is similar to the picture that emerged from lineage analyses performed in the vertebrate spinal cord showing that cell lineages are not dedicated to the production of motor neurons (Leber et al., 1990). As in the fly, closely related cells in the spinal cord may have distinct fates. Conversely, although adult fly motor neurons are born from stereotyped lineages, position within the CNS determines NB identities and, consequently, the progeny they generate (Bhat, 1998). Although C. elegans has a more extreme version of a lineagebased mechanism, even in this case cell-cell signaling plays an important role in specifying identities (Schnabel, 1991, 1997). These considerations blur the distinction between lineage and position-based mechanisms and suggest that both play a role in vertebrates and invertebrates.

Consistent with the idea that lineage may play a role in vertebrates, the transcription factor Coup-TF acts as a temporal switch between neurogenesis and gliogenesis in the vertebrate brain (Naka et al., 2008). Interestingly, Coup-TF is a relative of Drosophila svp, which encodes one of the temporal transcription factors used in postembryonic fly neuroblasts (Maurange et al., 2008). The use of Coup-TF/Svp for executing a temporal switch in both flies and vertebrates suggests the existence of a conserved molecular mechanism for controlling developmental timing in neural lineages (Jacob et al., 2008; Naka et al., 2008).

\section{Developmental logic in forming neural circuits}

Because motor neurons receive complex inputs from interneurons and sensory neurons, the architecture of their dendritic arbors is critical for forming the circuitry that is required for locomotion. Our initial analysis of the dendritic arbors of the leg motor neurons suggests that, as in other systems (Marin et al., 2002; Wong et al., 2002; Landgraf et al., 2003), they exhibit a functional organization in the thoracic neuromere. For example, nine leg motor neurons, targeting two different reductor muscles in different leg segments (coxa and femur), have overlapping dendritic arbors (Fig. 7D). That these nine motor neurons have similar dendritic architectures suggests that they share presynaptic inputs, perhaps allowing these two reductor muscles to contract in synchrony. Similarly, all eight motor neurons that have dendrites that cross the midline of the CNS, and thus probably make contacts with neurons in the contralateral neuromere, send their axons to one of two long tendon muscles, one in the tibia and one in the femur. These two examples suggest that the organization of motor neuron dendrites may be important for muscle synergies as described in vertebrate locomotion (Drew et al., 2008).

In vertebrate motor systems, motor neuron cell bodies are organized in columns and pools that correlate with their muscle targets (Landmesser, 1978). This organization implies that many of the presynaptic inputs into the motor neurons within individ- ual pools will be similar. Consistently, in some cases, the dendritic arbors of motor neurons have been shown to correlate with motor neuron targeting (Vrieseling and Arber, 2006). In these examples, the arborization patterns are controlled by the transcription factor Pea3, which requires a specific Hox code to be activated, but is only induced after motor axons invade the limb target (Dasen et al., 2005; Vrieseling and Arber, 2006). In contrast, the myotopic map exhibited by the dendrites of the fly larval motor neurons does not need target muscles to form (Landgraf et al., 2003). In the fly olfactory system, the dendrites of projection neurons form a map in the antennal lobe before the arrival of olfactory receptor neurons (ORNs), suggesting that this map forms independently of ORNs (Komiyama and Luo, 2007). It remains unclear whether the characteristic dendritic arbors of the fly's leg motor neurons require muscle targeting or whether they form independently of their targets using local cues in the CNS and the identities they acquire at birth.

\section{References}

Bässler U, Büschges A (1998) Pattern generation for stick insect walking movements: multisensory control of a locomotor program. Brain Res Brain Res Rev 27:65-88.

Bhat KM (1998) Cell-cell signaling during neurogenesis: some answers and many questions. Int J Dev Biol 42:127-139.

Broadus J, Skeath JB, Spana EP, Bossing T, Technau G, Doe CQ (1995) New neuroblast markers and the origin of the aCC/pCC neurons in the Drosophila central nervous system. Mech Dev 53:393-402.

Brody T, Odenwald WF (2005) Regulation of temporal identities during Drosophila neuroblast lineage development. Curr Opin Cell Biol 17:672-675.

Burrows M (1992) Local circuits for the control of leg movements in an insect. Trends Neurosci 15:226-232.

Burrows M (1996) The neurobiology of an insect brain. New York: Oxford UP.

Büschges A, Akay T, Gabriel JP, Schmidt J (2008) Organizing network action for locomotion: insights from studying insect walking. Brain Res Rev 57:162-171.

Dasen JS, Tice BC, Brenner-Morton S, Jessell TM (2005) A Hox regulatory network establishes motor neuron pool identity and target-muscle connectivity. Cell 123:477-491.

Delcomyn F (1989) Walking in the American cockroach: the timing of motor activity in the legs during straight walking. Biol Cybern 60:373-384.

Doe CQ (2006) Chinmo and neuroblast temporal identity. Cell $127: 254-256$.

Doe CQ, Skeath JB (1996) Neurogenesis in the insect central nervous system. Curr Opin Neurobiol 6:18-24.

Drew T, Kalaska J, Krouchev N (2008) Muscle synergies during locomotion in the cat: a model for motor cortex control. J Physiol 586:1239-1245.

Estella C, Mann RS (2008) Logic of Wg and Dpp induction of distal and medial fates in the Drosophila leg. Development 135:627-636.

Estella C, McKay DJ, Mann RS (2008) Molecular integration of wingless, decapentaplegic, and autoregulatory inputs into Distalless during Drosophila leg development. Dev Cell 14:86-96.

Feng G, Laskowski MB, Feldheim DA, Wang H, Lewis R, Frisen J, Flanagan JG, Sanes JR (2000) Roles for ephrins in positionally selective synaptogenesis between motor neurons and muscle fibers. Neuron 25:295-306.

Ito K, Awasaki T (2008) Clonal unit architecture of the adult fly brain. Adv Exp Med Biol 628:137-158.

Jacob J, Maurange C, Gould AP (2008) Temporal control of neuronal diversity: common regulatory principles in insects and vertebrates? Development 135:3481-3489.

Jan LY, Jan YN (1976) L-glutamate as an excitatory transmitter at the Drosophila larval neuromuscular junction. J Physiol 262:215-236.

Jefferis GS, Marin EC, Stocker RF, Luo L (2001) Target neuron prespecification in the olfactory map of Drosophila. Nature 414:204-208.

Jessell TM (2000) Neuronal specification in the spinal cord: inductive signals and transcriptional codes. Nat Rev Genet 1:20-29.

Komiyama T, Luo L (2007) Intrinsic control of precise dendritic targeting by an ensemble of transcription factors. Curr Biol 17:278-285.

Landgraf M, Jeffrey V, Fujioka M, Jaynes JB, Bate M (2003) Embryonic 
origins of a motor system: motor dendrites form a myotopic map in Drosophila. PLoS Biol 1:E41.

Landmesser L (1978) The distribution of motoneurones supplying chick hind limb muscles. J Physiol 284:371-389.

Laskowski MB, Sanes JR (1987) Topographic mapping of motor pools onto skeletal muscles. J Neurosci 7:252-260.

Leber SM, Breedlove SM, Sanes JR (1990) Lineage, arrangement, and death of clonally related motoneurons in chick spinal cord. J Neurosci 10:2451-2462.

Lee T, Luo L (1999) Mosaic analysis with a repressible cell marker for studies of gene function in neuronal morphogenesis. Neuron 22:451-461.

Lee T, Lee A, Luo L (1999) Development of the Drosophila mushroom bodies: sequential generation of three distinct types of neurons from a neuroblast. Development 126:4065-4076.

Mahr A, Aberle H (2006) The expression pattern of the Drosophila vesicular glutamate transporter: a marker protein for motoneurons and glutamatergic centers in the brain. Gene Expr Patterns 6:299-309.

Maqbool T, Jagla K (2007) Genetic control of muscle development: learning from Drosophila. J Muscle Res Cell Motil 28:397-407.

Marin EC, Jefferis GS, Komiyama T, Zhu H, Luo L (2002) Representation of the glomerular olfactory map in the Drosophila brain. Cell 109:243-255.

Maurange C, Gould AP (2005) Brainy but not too brainy: starting and stopping neuroblast divisions in Drosophila. Trends Neurosci 28:30-36.

Maurange C, Cheng L, Gould AP (2008) Temporal transcription factors and their targets schedule the end of neural proliferation in Drosophila. Cell 133:891-902.

McKay DJ, Estella C, Mann RS (2009) The origins of the Drosophila leg revealed by the cis-regulatory architecture of the Distalless gene. Development 136:61-71.

Morata G (2001) How Drosophila appendages develop. Nat Rev Mol Cell Biol 2:89-97.

Naka H, Nakamura S, Shimazaki T, Okano H (2008) Requirement for COUP-TFI and II in the temporal specification of neural stem cells in CNS development. Nat Neurosci 11:1014-1023.
Pearson BJ, Doe CQ (2004) Specification of temporal identity in the developing nervous system. Annu Rev Cell Dev Biol 20:619-647.

Prokop A, Technau GM (1991) The origin of postembryonic neuroblasts in the ventral nerve cord of Drosophila melanogaster. Development 111:79-88.

Schnabel R (1991) Early determinative events in Caenorhabditis elegans. Curr Opin Genet Dev 1:179-184.

Schnabel R (1997) Why does a nematode have an invariant cell lineage? Semin Cell Dev Biol 8:341-349.

Soler C, Daczewska M, Da Ponte JP, Dastugue B, Jagla K (2004) Coordinated development of muscles and tendons of the Drosophila leg. Development 131:6041-6051

Sulston JE (1976) Post-embryonic development in the ventral cord of Caenorhabditis elegans. Philos Trans R Soc Lond B Biol Sci 275:287-297.

Sulston JE (1983) Neuronal cell lineages in the nematode Caenorhabditis elegans. Cold Spring Harb Symp Quant Biol 48:443-452.

Sulston JE, Horvitz HR (1977) Post-embryonic cell lineages of the nematode, Caenorhabditis elegans. Dev Biol 56:110-156.

Truman JW, Bate M (1988) Spatial and temporal patterns of neurogenesis in the central nervous system of Drosophila melanogaster. Dev Biol 125:145-157.

Truman JW, Schuppe H, Shepherd D, Williams DW (2004) Developmental architecture of adult-specific lineages in the ventral CNS of Drosophila. Development 131:5167-5184.

Tryba AK, Ritzmann RE (2000) Multi-joint coordination during walking and foothold searching in the Blaberus cockroach. II. Extensor motor neuron pattern. J Neurophysiol 83:3337-3350.

Tsuchida T, Ensini M, Morton SB, Baldassare M, Edlund T, Jessell TM, Pfaff SL (1994) Topographic organization of embryonic motor neurons defined by expression of LIM homeobox genes. Cell 79:957-970.

Vrieseling E, Arber S (2006) Target-induced transcriptional control of dendritic patterning and connectivity in motor neurons by the ETS gene Pea3. Cell 127:1439-1452.

Wong AM, Wang JW, Axel R (2002) Spatial representation of the glomerular map in the Drosophila protocerebrum. Cell 109:229-241. 